\title{
Hepatotoxic pyrrolizidine alkaloids induce DNA damage response in rat liver in a 28-day feeding study
}

\author{
Johanna Ebmeyer ${ }^{1}$. Josef Daniel Rasinger ${ }^{2}$. Jan G. Hengstler ${ }^{3}$. Dirk Schaudien ${ }^{4}$. Otto Creutzenberg ${ }^{4}$. \\ Alfonso Lampen ${ }^{1} \cdot$ Albert Braeuning $^{1} \cdot$ Stefanie Hessel-Pras ${ }^{1}[$ (])
}

Received: 21 February 2020 / Accepted: 5 May 2020 / Published online: 17 May 2020

(c) The Author(s) 2020

\begin{abstract}
Pyrrolizidine alkaloids (PA) are secondary plant metabolites that occur as food and feed contaminants. Acute and subacute PA poisoning can lead to severe liver damage in humans and animals, comprising liver pain, hepatomegaly and the development of ascites due to occlusion of the hepatic sinusoids (veno-occlusive disease). Chronic exposure to low levels of PA can induce liver cirrhosis and liver cancer. However, it is not well understood which transcriptional changes are induced by PA and whether all hepatotoxic PA, regardless of their structure, induce similar responses. Therefore, a 28-day subacute rat feeding study was performed with six structurally different PA heliotrine, echimidine, lasiocarpine, senecionine, senkirkine, and platyphylline, administered at not acutely toxic doses from 0.1 to $3.3 \mathrm{mg} / \mathrm{kg}$ body weight. This dose range is relevant for humans, since consumption of contaminated tea may result in doses of $\sim \rho \mu \mathrm{g} / \mathrm{kg}$ in adults and cases of PA ingestion by contaminated food was reported for infants with doses up to $3 \mathrm{mg} / \mathrm{kg}$ body weight. ALT and AST were not increased in all treatment groups. Whole-genome microarray analyses revealed pronounced effects on gene expression in the high-dose treatment groups resulting in a set of 36 commonly regulated genes. However, platyphylline, the only 1,2-saturated and, therefore, presumably non-hepatotoxic PA, did not induce significant expression changes. Biological functions identified to be affected by high-dose treatments $(3.3 \mathrm{mg} / \mathrm{kg}$ body weight) comprise cell-cycle regulation associated with DNA damage response. These functions were found to be affected by all analyzed 1,2-unsaturated PA.

In conclusion, 1,2-unsaturated hepatotoxic PA induced cell cycle regulation processes associated with DNA damage response. Similar effects were observed for all hepatotoxic PA. Effects were observed in a dose range inducing no histopathological alterations and no increase in liver enzymes. Therefore, transcriptomics studies identified changes in expression of genes known to be involved in response to genotoxic compounds at PA doses relevant to humans under worst case exposure scenarios.
\end{abstract}

Keywords Pyrrolizidine alkaloids $\cdot$ Hepatotoxicity $\cdot$ Transcriptomics $\cdot$ DNA damage

\section{Abbreviations}

ALT Alanine aminotransferase

ANOVA Analysis of variance

Albert Braeuning and Stefanie Hessel-Pras equally contributed

Electronic supplementary material The online version of this article (https://doi.org/10.1007/s00204-020-02779-2) contains supplementary material, which is available to authorized users.

Stefanie Hessel-Pras

stefanie.hessel-pras@bfr.bund.de

1 German Federal Institute for Risk Assessment, Max-Dohrn-Str. 8-10, 10589 Berlin, Germany

2 Institute of Marine Research (IMR), Postboks 1870 Nordnes, NO-5817, Bergen, Norway
AST Aspartate aminotransferase

bw Body weight

CYP Cytochrome P450 monooxygenase(s)

IPA Ingenuity pathway analysis

qPCR Quantitative real-time PCR

PA Pyrrolizidine alkaloid(s)

PCA Principal component analysis

3 Leibniz Research Centre for Working Environment and Human Factors, Technical University Dortmund, Ardeystr. 67, 44139 Dortmund, Germany

4 Fraunhofer Institute for Toxicology and Experimental Medicine ITEM, Nikolai-Fuchs-Straße 1, 30625 Hanover, Germany 


\section{Introduction}

Pyrrolizidine alkaloids (PA) are a group of secondary plant metabolites that belong to the most widely distributed natural toxins. PA can contaminate food and feed and, thus, may affect health of consumers, livestock and wildlife (Stegelmeier et al. 1999; Wiedenfeld 2011). In Germany and Europe, tea, honey and herbal spices were identified as the main sources contributing to human exposure to PA (BfR 2018; BfR 2019; Bodi et al. 2014; EFSA 2017; Mulder et al. 2018). The European Food Safety Authority and the German Federal Institute for Risk Assessment concluded that uptake of contaminated food from the European Market may pose a risk to human health, especially for people consuming high amounts of these products, and for children (BfR 2018; BfR 2019; EFSA 2017).

PA share a common basic structure. They consist of a 1-hydroxymethylpyrrolizidine (necine base) esterified with one or two aliphatic mono- or dicarboxylic acids, socalled necine acids. According to their necine base, they are divided into four different structure types: retronecine-, heliotridine-, otonecine- and platynecine-type PA. Furthermore, they can be grouped according to their degree of esterification into monoesters, non-cyclic diesters and cyclic diesters. Due to different combinations of different necine bases and necine acids, a wide variety of structurally different PA congeners exists. Today, several hundred PA are known; probably produced by more than 6000 plant species (Stegelmeier et al. 1999). Oxidation of the nitrogen atom at one bridgehead of the two fused pentagonal carbon rings leads to PA $N$-oxides. PA $N$-oxides show higher water solubility compared to the free bases, and, thus, the $N$-oxide type is the predominating form in plants (Wiedenfeld et al. 2008). Since PA $N$-oxides can be reduced to the free base form, it is considered that PA $N$-oxides and free PA exert comparable toxicity (Wiedenfeld et al. 2008).
Figure 1 summarizes the structure of the PA representatives used in this study and assigns them to their respective structure type.

While the retronecine-, heliotridine- and otonecinetype PA are based on a 1,2-unsaturated necine base, the platynecine type lacks this double bond in 1,2-position. The 1,2-double bond is required for toxicity. Therefore, platynecine-type PA are assumed to be non-toxic. Further requirements for toxicity are described as follows: (i) at least one hydroxyl group needs to be attached to the pyrrolizidine ring via one carbon atom, (ii) at least one of this hydroxyl groups needs to be esterified, and (iii) the acid moiety needs to have a branched chain (Mattocks 1986). Toxicity is assumed to increase from monoesters to non-cyclic diesters and finally cyclic diesters with heliotridine-type PA being more toxic compared to retronecine-type PA (Merz and Schrenk 2016). It is considered that toxicity is not based on the PA parent compounds themselves but on their metabolites formed during bioactivation reactions located mainly in the liver. Hepatic metabolism is initiated by a cytochrome $\mathrm{P} 450$ (CYP)-mediated oxidation followed by spontaneous dehydration. Resulting pyrrolic esters are highly reactive and able to form protein and DNA adducts as well as DNA/protein cross-links which are assumed to be responsible for toxicity (Mattocks 1986; Ruan et al. 2014; Wiedenfeld et al. 2008).

Consumption of PA-contaminated food may lead to severe liver failure. Several case studies report the development of veno-occlusive disease after uptake of highly PA-contaminated wheat flour, seeds or herbal tea (Bras et al. 1954; Datta et al. 1978; Kakar et al. 2010; Mohabbat et al. 1976; Tandon et al. 1976). Furthermore, genotoxic, carcinogenic and pneumotoxic properties of PA have been described (Culvenor et al. 1969; Fu et al. 2004; Moreira et al. 2018). Some studies also point to the development of cholestasis (Hessel-Pras et al. 2020; Luckert et al. 2015). However, most of these specific effects were observed in the high-dose range. Our study aimed to identify toxic

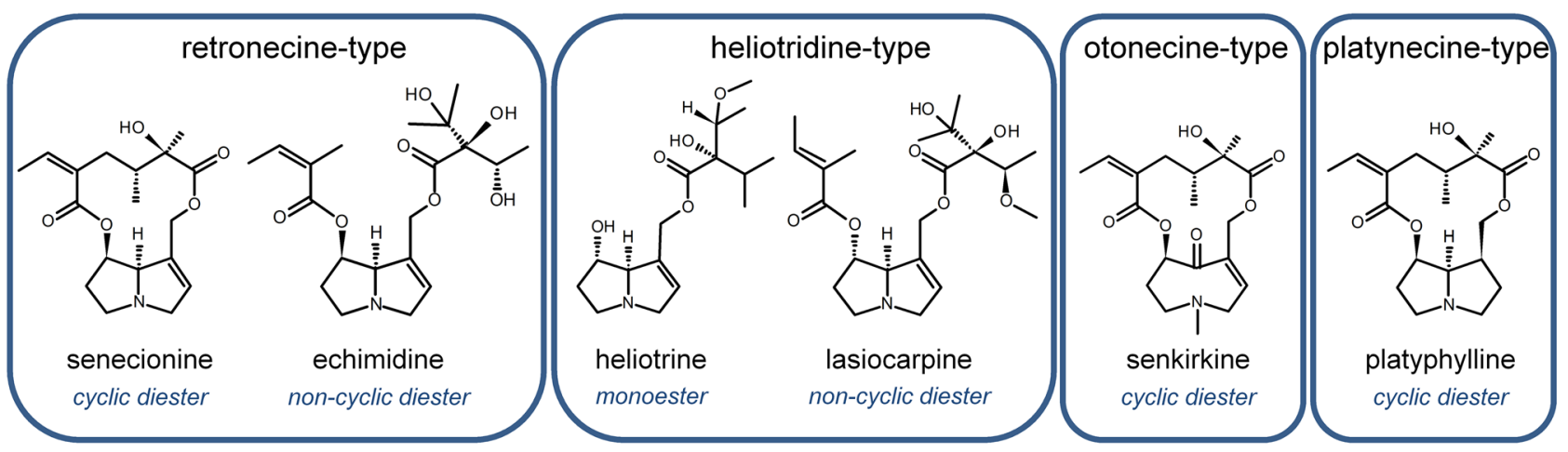

Fig. 1 Structure of the PA representatives senecionine, echimidine, heliotrine, lasiocarpine, senkirkine and platyphylline used in this study. These six PA representatives cover the different necine structure types as well as the different degrees of esterification. 
effects in a non-acute toxic dose range reflecting human exposure scenarios over a time period of 28 days to display repeated exposure and to achieve substantial responses on gene expression level in the low dose range. Furthermore, we wanted to test if structurally different PA affect different pathways or result in a similar toxicity pattern. Therefore, we chose a whole-genome transcriptomic approach in male Fischer rats treated by gavage with the PA representatives echimidine, heliotrine, lasiocarpine, senecionine, senkirkine and platyphylline covering all existing necine base structure types as well as monoesters, non-cyclic and cyclic diesters. Platyphylline is considered to be non-hepatotoxic due to the missing 1,2-double bond and was, therefore, included as a non-toxic control.

\section{Materials and methods}

\section{Chemicals}

Echimidine (94\% purity), heliotrine (91\% purity), lasiocarpine (98\% purity), senecionine (99\% purity) and senkirkine (98\% purity) were purchased from PhytoLab (Verstenbergsgreuth, Germany). Platyphylline (95\% purity) was purchased from BOC Sciences (New York, New York, USA). Senecionine was dissolved in 0.2-M hydrochloric acid. Afterwards, $\mathrm{pH}$ was adjusted to 6-7 using 0.2-M sodium hydroxide solution. Sodium chloride $(\mathrm{NaCl})$ was added to obtain a solution containing $0.15-\mathrm{M} \mathrm{NaCl}$. All other PA were dissolved in $0.15-\mathrm{M}$ aqueous $\mathrm{NaCl}$ solution.

\section{Animal experiments}

A 28-day oral toxicity study was performed with male Fischer rats (strain F-344/DuCrl) exposed to six PA, namely echimidine, heliotrine, lasiocarpine, senecionine, senkirkine and platyphylline at the Fraunhofer Institute for Toxicology and Experimental Medicine (ITEM, Hanover, Germany). All animal experiments were conducted in compliance with the regulation of the German Animal Protection Law, following the principles of Good Laboratory Practice (OECD 1998) and considering OECD Guideline 407 for repeated dose 28-day oral toxicity study in rodents (adopted guideline; October 3, 2008).

A total of 128 male Fischer rats (strain F-344/DuCrl) were purchased from Charles River Deutschland (Sulzfeld, Germany) and divided into 25 groups. Grouping was performed randomly according to the average body weight of the animals resulting in groups with the same average body weight. Each treatment group consisted of five animals. Vehicle control included 8 animals. Following delivery, the 4-5-week-old animals were allowed to acclimatize for 4 weeks; at study start (i.e., at an age of 8-9 weeks), body weights of about $200 \mathrm{~g}$ were determined. Animals were group-housed in type IV Makrolon ${ }^{\circledR}$ (polycarbonate) cages and maintained under conventional laboratory conditions. The temperature $\left(22 \pm 2{ }^{\circ} \mathrm{C}\right)$ and the relative humidity $(55 \pm 15 \%)$ of the animal room were monitored and recorded continuously. A 12-h light/dark cycle was used. Rats had access to commercial diet (Ssniff V1534, SsniffSpezialdiäten, Soest, Germany) and tap water ad libitum. Animals were treated for 28 days daily by gavage with a flexible tube. A solution of $0.15-\mathrm{M} \mathrm{NaCl}$ served as vehicle control (group 1). Groups 2-25 were treated with 0.1, 0.33, 1.0 , or $3.3 \mathrm{mg} / \mathrm{kg}$ body weight (bw) of single PA. Information about animal treatment is summarized in Table 1. Doses were chosen following a two-week and 13-week NTP study with riddelliine in Fischer rats (National Institutes of Health 1993). According to the publication of Merz and Schrenk (2016), the cyclic retronecine-type diester riddelliine exhibits comparable potency like senecionine which was used in this study. A dose of $3.3 \mathrm{mg} / \mathrm{kg}$ bw was chosen as the highest dose to avoid unspecific effects resulting from general toxicity.

Throughout the study, individual body weight was recorded once a week. Additionally, animals were observed daily for clinical symptoms. One day after the last administration, animals were killed and prepared for histopathological examination of lungs and livers, blood analysis and transcriptomic analysis. Animals were killed by a lethal dose of pentobarbital. A total of approximately $3-\mathrm{ml}$ heparinized blood (+ Nembutal) was collected from the vena cava caudalis before start of whole-body perfusion for liver preparation. Liver weights were determined.

\section{Histopathological examination and analysis of transaminases in blood}

One slice with a maximal diameter of $7 \mathrm{~mm}$ of the rat liver lobes 1 and 3 was fixed in buffered formalin (10\%), embedded in paraffin, sectioned, and stained with hematoxylin and eosin. Levels of the transaminases aspartate aminotransferase and alanine aminotransferase in blood were analyzed

Table 1 Summary of animal treatment

\begin{tabular}{lll}
\hline Substance & $\begin{array}{l}\text { Daily dose } \\
(\mathrm{mg} / \mathrm{kg} \text { bw) }\end{array}$ & $\begin{array}{l}\text { Num- } \\
\text { ber of } \\
\text { animals }\end{array}$ \\
\hline Vehicle control (0.15-M NaCl) & 0 & 8 \\
PA (echimidine, heliotrine, lasiocarpine, & 0.1 & 5 \\
senecionine, senkirkine, or platyphylline) & 0.33 & 5 \\
& 1.0 & 5 \\
& 3.3 & 5 \\
\hline
\end{tabular}


by kinetic UV test at $37{ }^{\circ} \mathrm{C}$ as described before (Bergmeyer 1983).

\section{Transcriptomics analysis}

For transcriptomics analysis of liver, RNA was extracted using the RNeasy Mini Kit (Qiagen, Hilden, Germany) in combination with QiaShredder (Qiagen, Hilden, Germany). Thus, small pieces of the deep-frozen liver lobe 1 were homogenized under liquid nitrogen and transferred to RLT buffer (Qiagen, Hilden, Germany) containing 1\% $\beta$-mercaptoethanol. Afterwards, the lysate was further homogenized using QiaShredder and the RNA was isolated using RNeasy Mini Kit according to the Manufacturer's instructions. Concentration and purity of total RNA was measured at a Tecan M200Pro spectrophotometer using a NanoQuant Plate at wavelengths of $260 \mathrm{~nm}$ and $280 \mathrm{~nm}$, and on an Agilent 2100 BioAnalyzer (Agilent, Santa Clara, California, USA). Only high-quality RNA with RNA integrity numbers (RIN) higher than 9 were used for microarray analysis. Microarray analysis was performed at Eurofins (Aarhus, Denmark) using Affymetrix GeneChip Rat ClariomD arrays (Thermo Fisher Scientific, Waltham, Massachusetts, USA). The raw data of this publication have been deposited in NCBI's Gene Expression Omnibus (Edgar et al. 2002) and are accessible through GEO Series accession number GSE149678.

\section{Verification of microarray results by qPCR}

Extracted RNA was analyzed by quantitative real-time PCR (qPCR) for the expression of the following genes to validate microarray results: Abcblb, Aldhlal, Ckap2, Fas, Clec $4 f$, Csf1r and Inmt. The first 4 genes were found to be significantly $(\mathrm{q}<0.05)$ upregulated in microarray analysis. The last three genes were significantly $(q<0.05)$ downregulated. The genes were randomly chosen among the genes that were dysregulated by at least 3 out of 5 toxic PA. Gapdh and Actb were used as housekeeping genes. Extraction of RNA was performed as described for transcriptomics analysis (see Sect. "Transcriptomics analysis"). Afterwards, cDNA synthesis was conducted with 1000-ng RNA using the High Capacity cDNA Reverse Transcription Kit according to the manufacturer's instructions (Applied Biosystems, Foster City, California, USA). qPCR was performed using Maxima SYBR Green/ROX qPCR Master Mix (Thermo Fisher Scientific, Waltham, Massachusetts, USA) with $1 \mu$ of cDNA and 300 pmol of the respective forward and reverse primers on a Stratagene Mx3005P instrument (Agilent Technologies, Santa Clara, California, USA). Gene names, gene abbreviations, and used primer sequences are summarized in Table 2. All primers were designed using Primer 3 software (Rozen and Skaletsky 2000). For thermal cycling procedure, an initial denaturation and activation of the hot start Taq polymerase at $95^{\circ} \mathrm{C}$ for $15 \mathrm{~min}$ was followed by 40 cycles consisting of denaturation $\left(95^{\circ} \mathrm{C}, 15 \mathrm{~s}\right)$ and annealing and elongation $\left(60{ }^{\circ} \mathrm{C}, 1 \mathrm{~min}\right)$. Finally, final elongation was performed for $15 \mathrm{~min}$ at $60^{\circ} \mathrm{C}$. Additionally, a dissociation curve step was added.

\section{Statistical and bioinformatic data analysis}

For statistical analysis, microarray data were normalized using the robust multi-array-average (RMA) function of the limma (linear models for microarray and RNA-Seq data) package (Smyth et al. 2019, page 104) in R (R Core Team 2017; Ritchie et al. 2015) to assure that any batch effects between arrays are being removed. Subsequently, the dataset was filtered and only transcripts were kept for which annotations were available. The reduced dataset
Table 2 Primer sequences used in this study

\begin{tabular}{lll}
\hline Gene $^{1}$ & Sequence $\left(5^{\prime}-3^{\prime}\right)$ & \\
\cline { 2 - 3 } & Forward primer & Reverse primer \\
\hline Abcblb & TGGCCATGTACGCCTACTATTACA & AAAACCGGCTGAAAATGTCGTT \\
Actb & TGTGTTGTCCTGTATGCCT & AGCGCGTAACCCTCATAGAT \\
Aldhlal & GATGCCGACTTGGACATTGC & GACGCAGCATTGGCCTTGAT \\
Ckap2 & TAATGCCAGGCTGACAGGAA & GCTGTGGTATCTTTGGGCTG \\
Clec4f & ATGAAGGAGGCGGAACTGAA & ATGGGAGCAGCTGACTTAGG \\
Csflr & CAAACTCCACCTGAACCGTG & TATCGCAGGGTGAGCTCAAA \\
Fas & ACATCTGGAGAACTGCCGAA & CATCGATCTTGCTTTCCGGG \\
Gapdh & CCGTGGGGCAGCCCAGAA & GCCCCAGCATCAAAGGTGGAGGA \\
Inmt & ACTAGAATGGCAGGCAAGGT & GCTGTAGAAGGTGGTCAGGT \\
\hline
\end{tabular}

${ }^{1}$ Gene names: Abcbl ATP-binding cassette subfamily B member 1, Actb actin beta, Aldhlal aldehyde dehydrogenase 1 family member A1, Ckap2 cytoskeleton-associated protein 2, Clec4 C-type lectin domain family 4, Csf1r colony stimulating factor 1 receptor, Fas fas cell surface death receptor, Gapdh glyceraldehyde-3-phosphate dehydrogenase, Inmt indolethylamine N-methyltransferase 
comprising 26,480 probe sets was analyzed further using Qlucore Omics Explorer (version 3.3, Qlucore, Lund, Sweden). The short technical note on the use of Qlucore for omics data mining gives a short summary of how the analysis workflow employed in the present study has been used to reduce large number of altered genes to a few robust discriminative features (Rasinger and Lie). After a correction for batch-dependent differences of microarray chips, a comparison of all high-dose groups of single PA was performed using one-way analysis of variance (ANOVA). Fold changes for significantly dysregulated genes $(q<0.05)$ were determined using two-group comparison tests against vehicle control. The $q$-values are Benjamini-Hochberg-corrected p-values as outputted by Qlucore (Benjamini and Hochberg 1995) to control the false discovery rate. In addition, rank regressions were performed across all doses for each individual PA.

Following statistical analysis, data was subjected to Ingenuity Pathway Analysis (IPA, version 48,207,413, Qiagen Bioinformatics, Redwood City, California, USA). Comparison analyses to predict affected canonical pathways, diseases and functions and upstream regulators were performed.

Data regarding body weight, relative liver weight, levels of transaminases were recorded and analyzed by one-way ANOVA followed by Dunnett's post hoc test using the PROVANTIS system (version 8.4.3.1). Relative gene expression as analyzed by qPCR was statistically analyzed by one-way ANOVA followed by Dunnett's post hoc test using SigmaPlot software.

\section{Results}

\section{Body weights, organ weights and transaminases in blood}

No differences in body weight development of treated animals compared to the vehicle control were observed. Individual data are summarized in supplementary data Figure S1. Absolute liver weights did also not show relevant differences between vehicle control and treatment groups. The high-dose senkirkine treatment group showed a slightly but significantly increased relative liver weight (111\% of vehicle control, $p<0.05$ ) (Supplementary data, Fig. S2).

The levels of the hepatic enzymes aspartate aminotransferase (AST) and alanine aminotransferase (ALT) were determined in blood to identify hepatotoxicity. All groups did not show statistically significant $(p<0.05)$ changes in neither ALT nor AST levels, except the high-dose group of lasiocarpine $(3.3 \mathrm{mg} / \mathrm{kg}$ bw) which showed a statistically significant $(p<0.05)$ increase of ALT from 85.8 $\pm 22.5 \mathrm{U} / 1$ in control animals to $160.5 \pm 19.4 \mathrm{U} / \mathrm{l}$ (Supplementary data
Fig. S3). However, no morphological changes were detected in this group during histopathological examination (see "Histopathology").

\section{Histopathology}

The livers of all animals were examined histopathologically. A (multi)focal random mixed inflammatory cell infiltration as well as (multi)focal periportal mononuclear cell infiltration were seen to a very slight extent in up to two animals per group in most groups (Supplementary data Fig. S4). Within the senecionine high-dose group $(3.3 \mathrm{mg} / \mathrm{kg} \mathrm{bw})$, four animals showed a (multi)focal random mixed inflammatory cell infiltration (3/5 very slight and 1/5 slight). However, these lesions occur occasionally in rats and are considered to be unrelated to the treatment.

Taken together, results of biochemical analysis showed only weak effects for lasiocarpine in the highest dose group by inducing a twofold increase in ALT activity. This suggests that the chosen dose range represents doses in the borderline range for classic toxicity and, thus, may be in the range of or below the no-observed adverse effect levels (NOAEL). Therefore, it is assumed that the changes in the following gene expression analysis are PA-specific effects instead of unspecific effects resulting from massive injury of the whole organism.

\section{Whole-genome microarray analysis}

A whole-genome microarray analysis was performed to identify PA-affected pathways in dependence of the PA structure type. For statistical evaluation, the whole dataset including 70,580 probe sets was reduced to 26,480 annotated probe sets and an ANOVA between all high-dose groups and vehicle control was performed. This resulted in 284 significantly altered $(q<0.05)$ probe sets (see Supplementary data, Table S1). Hierarchical clustering of these transcripts according to the PA exposure groups is shown in Fig. 2a.

Platyphylline as a non-hepatotoxic PA showed a gene expression pattern very similar to the vehicle control up to the highest tested dose of $3.3 \mathrm{mg} / \mathrm{kg}$ bw. Senkirkine showed the strongest alterations compared to the vehicle control, followed by senecionine. Heliotrine and lasiocarpine showed alterations of moderate magnitude between platyphylline and senecionine/senkirkine. Echimidine was the treatment group with the highest variability in the magnitude of alterations between the different individuals. To identify the direction of regulation, significantly differentially regulated $(q<0.05$, ANOVA) probe sets were analyzed further performing two-group comparisons of each PA against the vehicle control. The number of up- and downregulated probe sets is shown in Fig. 2b. Most of the dysregulated genes were upregulated. Again, senecionine and senkirkine showed the 
Fig. 2 Comparison of dysregulated probe sets after oral treatment of male Fischer rats by gavage for 28 days with six different PA. Results for the high dose groups $(3.3 \mathrm{mg} / \mathrm{kg} \mathrm{bw})$ are presented. a Clustering of gene expression changes in the liver following exposure to PA. Gene expression profiles were analyzed using Affymetrix microarrays. Rats were exposed to either $3.3 \mathrm{mg} / \mathrm{kg}$ bw echimidine (yellow), heliotrine (pink), lasiocarpine (red), senecionine (light blue), senkirkine (green), platyphylline (orange) or a solution of $0.15 \mathrm{M} \mathrm{NaCl}$ serving as vehicle control (dark blue). Each column represents a single animal. Each row shows a specific transcript. Microarray data were normalized, batch corrected and statistically analyzed using Qlucore Omics Explorer. A total of 284 probe sets were identified to be significantly differentially regulated $(q<0.05$, ANOVA). Individual PA exposure effects and fold changes were determined by performing two-group comparison test versus the vehicle control. b The bar chart shows the average numbers of up- and downregulated probe sets for each treatment group. $\mathbf{c}$ The Venn diagram shows the average number of dysregulated probe sets for the different treatment groups (high dose groups with $3.3 \mathrm{mg} / \mathrm{kg}$ bw only) and their overlap. The Venn diagram was generated using https://bioinformatics.psb.ugent .be/webtools/Venn/

highest numbers of significantly dysregulated probe sets. Platyphylline, a PA representative assumed to be non-hepatotoxic, provoked significant regulation of only 11 probe sets in total. The Venn diagram in Fig. 2c shows the overlap of similar dysregulated probe sets for the different PA. In total, a signature of 36 dysregulated probe sets corresponding to 35 individual genes was identified to be dysregulated by all hepatotoxic PA. This gene signature is summarized in Fig. 3. The genes regulated by individual PA are summarized in Table S2 in the supplemental material.

This list includes a high number of genes associated with DNA damage response; cell cycle control/integrity, or apoptosis: Rad51 recombinase (Rad51) plays an important role during homologous recombination and is upregulated. O-6-methylguanine-DNA methyltransferase $(M g m t)$ is involved in the repair of methylated DNA and is also upregulated. Cyclin-dependent kinase inhibitor 1A (Cdknla) and Cyclin G1 (Ccngl) function as regulators of the cell cycle. Cdknla regulates cell cycle progression and leads to cell cycle G1 phase arrest. Ccgnl is associated with DNA damage-induced G2/M phase arrest. Both genes are regulated by the tumor suppressor protein p53 and were upregulated in this study. Upregulation of these genes suggests that PA may provoke DNA damage and, thus, induce expression of genes important for DNA repair and subsequent cell cycle control. The transcript for DNA damage-induced apoptosis suppressor (Ddias) was found to be upregulated; this may indicate a possible counter-regulation elicited to protect against DNA damage-induced cell death. MYB proto-oncogene like $2(M y b l 2)$ is a transcription factor that regulates cell cycle progression. Deregulation of $M y b l 2$ expression is associated with cancer initiation and progression (Musa et al. 2017). Polo-like kinase 4 (Plk4) mediates duplication of centrioles during cell cycle and was also upregulated in

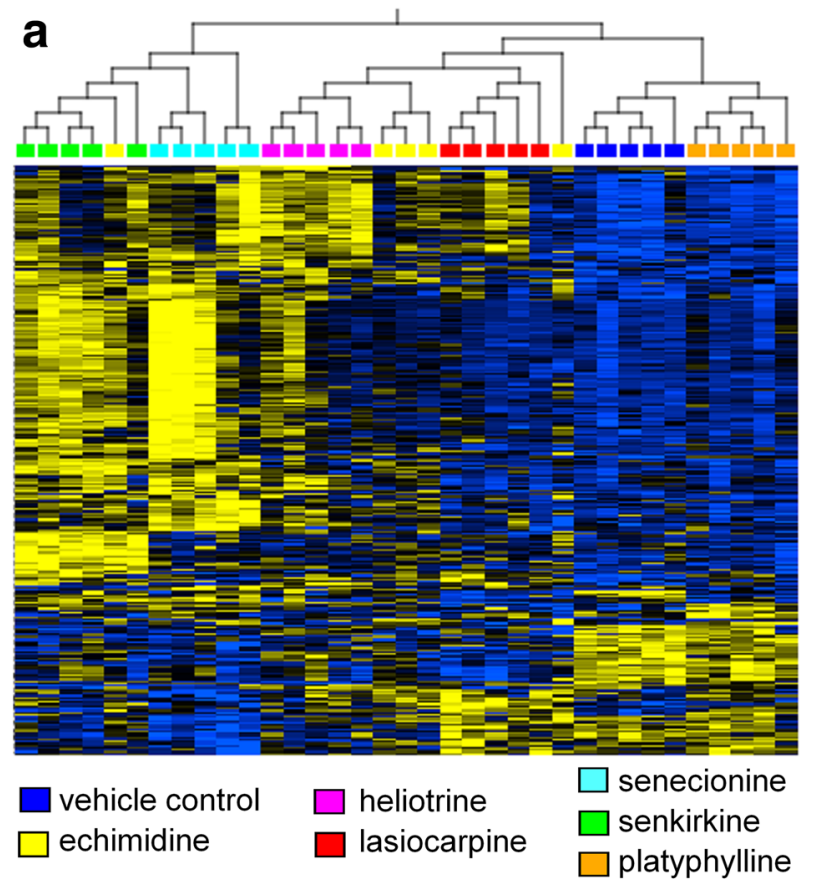

b

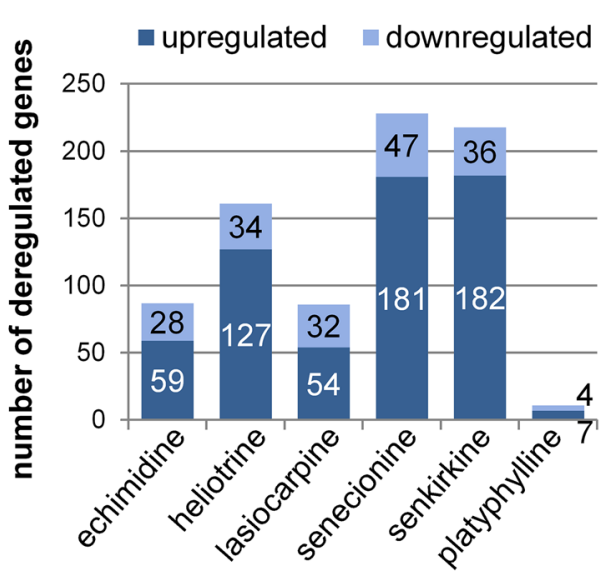

C

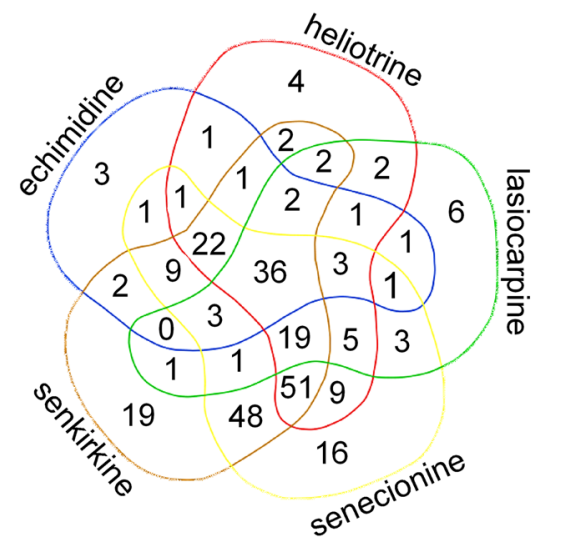




\begin{tabular}{|c|c|c|c|c|c|c|c|}
\hline $\begin{array}{l}\text { gene } \\
\text { symbol }\end{array}$ & gene name & echimidine & heliotrine & $\begin{array}{l}\text { fold change } \\
\text { lasiocarpine }\end{array}$ & senecionine & senkirkine & biological function \\
\hline Cfd & complement factor $\mathrm{D}$ & 0,77 & 0,75 & 0,76 & 0,74 & 0,75 & \\
\hline Aldh1a7 & aldehyde dehydrogenase family 1 , subfamily $A 7$ & 1,29 & 1,68 & 1,54 & 1,62 & 1,32 & xenobiotic metabolism \\
\hline Mok & MOK protein kinase & 1,27 & 1,49 & 1,40 & 1,32 & 1,35 & \\
\hline Aldh1a1 & aldehyde dehydrogenase 1 family member $A 1$ & 2,81 & 5,20 & 3,28 & 3,61 & 2,84 & xenobiotic metabolism \\
\hline Pm20d2 & peptidase M20 domain containing 2 & 0,78 & 0,89 & 0,87 & 0,79 & 0,80 & \\
\hline Gria3 & glutamate ionotropic receptor AMPA type subunit 3 & 1,45 & 1,99 & 1,46 & 1,55 & 1,69 & \\
\hline Rad51 & RAD51 recombinase & 1,18 & 1,25 & 1,17 & 1,44 & 1,34 & DNA repair \\
\hline Gzma & granzyme A & 0,70 & 0,80 & 0,63 & 0,58 & 0,56 & \\
\hline Gsta1 & glutathione S-transferase alpha 1 & 1,19 & 1,38 & 1,32 & 1,29 & 1,29 & xenobiotic metabolism \\
\hline Adam 8 & ADAM metallopeptidase domain 8 & 1,83 & 5,83 & 1,54 & 3,76 & 2,13 & \\
\hline Nr3c1 & nuclear receptor subfamily 3 group $C$ member 1 & 0,76 & 0,82 & 0,80 & 0,67 & 0,76 & \\
\hline Rp/19 & ribosomal protein L19 & 1,16 & 1,21 & 1,19 & 1,19 & 1,16 & cell cycle \\
\hline Mgmt & O-6-methylguanine-DNA methyltransferase & 1,58 & 2,00 & 1,74 & 1,75 & 1,73 & DNA repair \\
\hline Cdkn1a & cyclin dependent kinase inhibitor $1 \mathrm{~A}$ & 2,06 & 3,13 & 2,44 & 2,72 & 2,28 & cell cycle \\
\hline Fcna & ficolin A & 0,74 & 0,69 & 0,66 & 0,71 & 0,74 & \\
\hline Clec4f & C-type lectin domain family 4 member $F$ & 0,66 & 0,59 & 0,46 & 0,68 & 0,63 & \\
\hline Mkrn1 & makorin ring finger protein 1 & 0,89 & 0,90 & 0,88 & 0,90 & 0,85 & transcriptional activity \\
\hline Cong1 & cyclin G1 & 1,43 & 2,04 & 1,61 & 1,91 & 1,59 & cell cycle \\
\hline Fas & Fas cell surface death receptor & 1,40 & 1,64 & 1,43 & 1,65 & 1,42 & apoptosis \\
\hline Rnf144a & ring finger protein $144 \mathrm{~A}$ & 0,82 & 0,83 & 0,87 & 0,77 & 0,79 & transcriptional activity \\
\hline Stac3 & $\mathrm{SH} 3$ and cysteine rich domain 3 & 0,67 & 0,60 & 0,67 & 0,54 & 0,62 & \\
\hline$K d m 5 b$ & lysine demethylase $5 \mathrm{~B}$ & 0,83 & 0,86 & 0,78 & 0,85 & 0,88 & \\
\hline Uhrf1 & ubiquitin like with PHD and ring finger domains 1 & 1,18 & 1,18 & 1,16 & 1,33 & 1,34 & transcriptional activity \\
\hline Myb/2 & MYB proto-oncogene like 2 & 1,35 & 1,72 & 1,48 & 1,65 & 1,38 & cell cycle \\
\hline Hpgd & 15-hydroxyprostaglandin dehydrogenase & 0,66 & 0,71 & 0,67 & 0,69 & 0,71 & \\
\hline Ddias & DNA damage induced apoptosis suppressor & 1,33 & 1,48 & 1,31 & 2,08 & 1,59 & apoptosis \\
\hline Csf1r & colony stimulating factor 1 receptor & 0,61 & 0,62 & 0,46 & 0,61 & 0,65 & \\
\hline Dtl & denticleless E3 ubiquitin protein ligase homolog & 1,23 & 1,32 & 1,16 & 1,67 & 1,54 & \\
\hline Gsta1 & glutathione S-transferase alpha 1 & 1,17 & 1,31 & 1,28 & 1,21 & 1,25 & xenobiotic metabolism \\
\hline Plk4 & polo like kinase 4 & 1,27 & 1,22 & 1,08 & 1,68 & 1,41 & cell cycle \\
\hline Mcm5 & minichromosome maintenance complex component 5 & 1,29 & 1,33 & 1,10 & 1,72 & 1,50 & cell cycle \\
\hline Eda2r & ectodysplasin A2 receptor & 2,40 & 4,78 & 2,80 & 3,54 & 4,18 & apoptosis \\
\hline Phtf1 & putative homeodomain transcription factor 1 & 0,84 & 0,88 & 0,83 & 0,88 & 0,82 & transcriptional activity \\
\hline Aox4 & aldehyde oxidase 4 & 0,84 & 0,86 & 0,78 & 0,79 & 0,82 & \\
\hline Bax & BCL2 associated X, apoptosis regulator & 1,18 & 1,25 & 1,21 & 1,23 & 1,26 & apoptosis \\
\hline Ebpl & emopamil binding protein like & 1,14 & 1,12 & 1,19 & 1,12 & 1,21 & \\
\hline
\end{tabular}

Fig. 3 Fold change and classification of biological function of 36 probe sets corresponding to 35 individual genes that are commonly dysregulated by all 5 toxic PA according to microarray data analy- sis of livers of male Fischer rats orally treated daily for 28 days with $3.3 \mathrm{mg} / \mathrm{kg}$ bw of the respective PA this study. Minichromosome maintenance complex component $5(\mathrm{Mcm} 5)$ is important for DNA replication during $\mathrm{G} 1$ and $\mathrm{S}$ phases of the cell cycle and was identified to be upregulated. Ribosomal protein L19 (Rpl19) is part of the 60S subunit of the ribosome and, thus, is essential for protein synthesis. Rpl19 was also among the upregulated common dysregulated genes. Upregulation of these three genes may point to replacement proliferation as a consequence of cell death resulting from DNA damage. Induction of cell death is furthermore emphasized by upregulation of genes related to cell death: the Fas cell surface death receptor (Fas) is involved in initiation of extrinsic apoptosis. An induction of apoptosis was also reported to be mediated by the ectodysplasin A2 receptor (Eda2r) (Sinha and Chaudhary 2004). BCL2-associated X (Bax) protein is another pro-apoptotic factor. Furthermore, several genes associated with transcriptional activity were identified as commonly dysregulated genes: ring finger proteins makorin ring finger protein 1 (Mkrnl) and ring finger protein 144A (Rnfl44a) were downregulated, and ubiquitin like with PHD and ring finger domains 1 (Uhrfl) was upregulated. These three genes are associated with a novel class of zinc finger proteins that may act as transcriptional regulators. Putative homeodomain transcription factor 1 (Phtrfl) was downregulated. Finally, genes related to xenobiotic metabolism showed an altered expression upon treatment with PA: aldehyde dehydrogenase family 1 member A7 (Aldh1a7), aldehyde dehydrogenase 1 family member A1 (Aldhlal) and glutathione $S$-transferase alpha 1 (Gstal) encode enzymes for xenobiotic metabolism and were all upregulated in this study. A total of 48 genes were individually regulated by only one single PA (listed in Supplementary Table S2). While the low number of individual genes per PA does not allow concluding on specific mechanisms activated by only one of the test compounds, it is obvious that several genes are related to cell cycle, DNA repair or genotoxic responses, for example cyclin D1 (Ccnd1), platelet-derived growth factor C $(P d g f c)$, KiSS-1 metastasis suppressor (Kiss1), CD244 molecule (Cd244), caspase 12 (Casp12), or non-homologous end-joining factor 1 (Nhej1), respectively.

Figure 4 depicts the dose dependence of significantly deregulated $(q<0.05)$ transcripts for each PA in a principal component analysis (PCA) plot. The PCA shows that the highest dose $(3.3 \mathrm{mg} / \mathrm{kg}$ bw) differs clearly from vehicle control among the main discriminate axis (PC1). A tendency of gene expression alterations compared to vehicle control was also observed for the second highest dose $(1.0 \mathrm{mg} / \mathrm{kg} \mathrm{bw})$. These results suggest that $3.3 \mathrm{mg} / \mathrm{kg}$ 

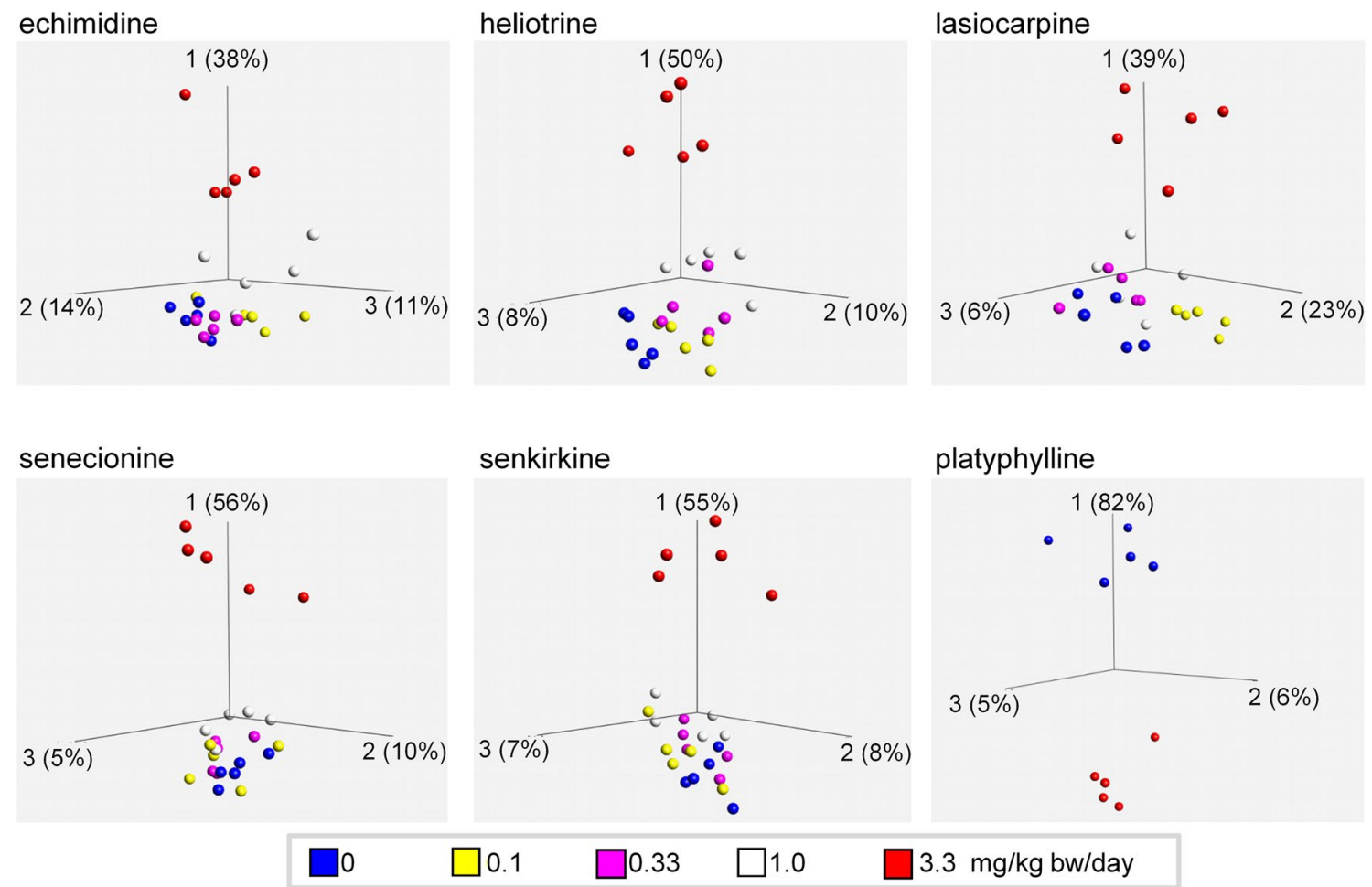

senkirkine

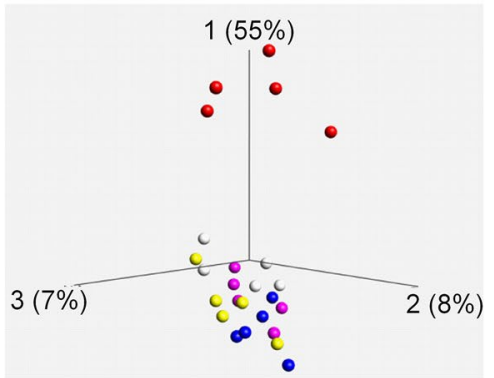

platyphylline

$\square 0.1$

0.33

1.0

$3.3 \mathrm{mg} / \mathrm{kg}$ bw/day

Fig. 4 Dose dependence of gene regulation for the different treatment groups. For each PA, all tested doses were analyzed by one-way ANOVA. Differentially regulated genes $(q<0.05)$ were subjected to principal component analyses (PCA). Differences in dose-related effects are shown in the PCA plots with each individual animal being represented by a single dot bw is a dose resulting in clear effects on the transcriptome of the liver, and that $1.0 \mathrm{mg} / \mathrm{kg}$ bw was in the borderline dose range near the NOEL for transcriptomic changes.

In case of platyphylline exposure, only the highest dose was analyzed. The PCA analysis of this group, therefore, displays a more pronounced discrimination along PC1, but of opposite direction compared to the other PA. Since unlike for the other PA no lower doses were analyzed, no conclusions concerning dose response can be drawn for this PA.

\section{Verification of microarray data}

The expression levels of 4 upregulated and 3 downregulated genes were additionally assessed by qPCR. The transcripts for $\mathrm{qPCR}$ validation were randomly chosen from the set of significantly dysregulated genes $(q<0.05)$ which were directly affected by at least 3 of all toxic PA in the highestdose group. The gene expression pattern as analyzed by qPCR was similar to that of the microarray assay (see Supplementary data Fig. S5), thus verifying the microarray data.

\section{Ingenuity Pathway Analysis}

IPA analysis was based on a subset of 284 significantly $(q<0.05$, ANOVA) dysregulated probe sets. For identification of a dose-dependent up- or downregulation of the transcripts, the correlation coefficient of a rank regression performed across all dose levels was uploaded to IPA. An IPA comparison analysis between all PA predicted affected canonical pathways, diseases and functions, and upstream regulators for the toxic PA echimidine, heliotrine, lasiocarpine, senecionine and senkirkine (Fig. 5). Platyphylline as a non-toxic PA representative showed no predicted activation or inhibition of any of the analyzed functions. The canonical pathway analysis predominantly predicted interaction with pathways related to DNA damage and cell cycle control/integrity (Fig. 5a).

IPA analysis found that ataxia telangiectasia mutated (ATM) signaling and tumor suppressor protein p53 signaling show the highest activation z-score. ATM signaling was predicted to be activated by heliotrine, senecionine and senkirkine; 553 signaling was predicted to be activated by all PA except platyphylline. According to the IPA knowledgebase (QIAGEN Inc., https://www.qiagenbioinform atics.com/products/ingenuity-pathway-analysis), both of these pathways are involved in initiation of DNA repair and able to lead to cell cycle arrest in the case of massive DNA damage. P53 furthermore regulates as a transcription factor the expression of several genes. Additionally, estrogenmediated S-phase entry (activated by heliotrine, senkirkine 
Fig. 5 Biological functions and pathways predicted to be activated or inactivated by PA according to Ingenuity Pathway Analysis (IPA). A set of 284 significantly $(q<0.05)$ dysregulated rat liver transcripts was analyzed with IPA. Canonical pathways (a), diseases and biofunctions (b) and hepatotoxic functions (c) are displayed. The heatmaps show activation z-scores for the respective canonical pathways. For diseases and biofunctions, the 30 diseases and functions with the highest positive or the lowest negative activation $\mathrm{z}$-scores are shown. Hepatotoxic functions were identified by IPA analysis for activation/inactivation of diseases and functions, filtered for hepatotoxicity. A complete list of affected functions is presented in the supplemental material
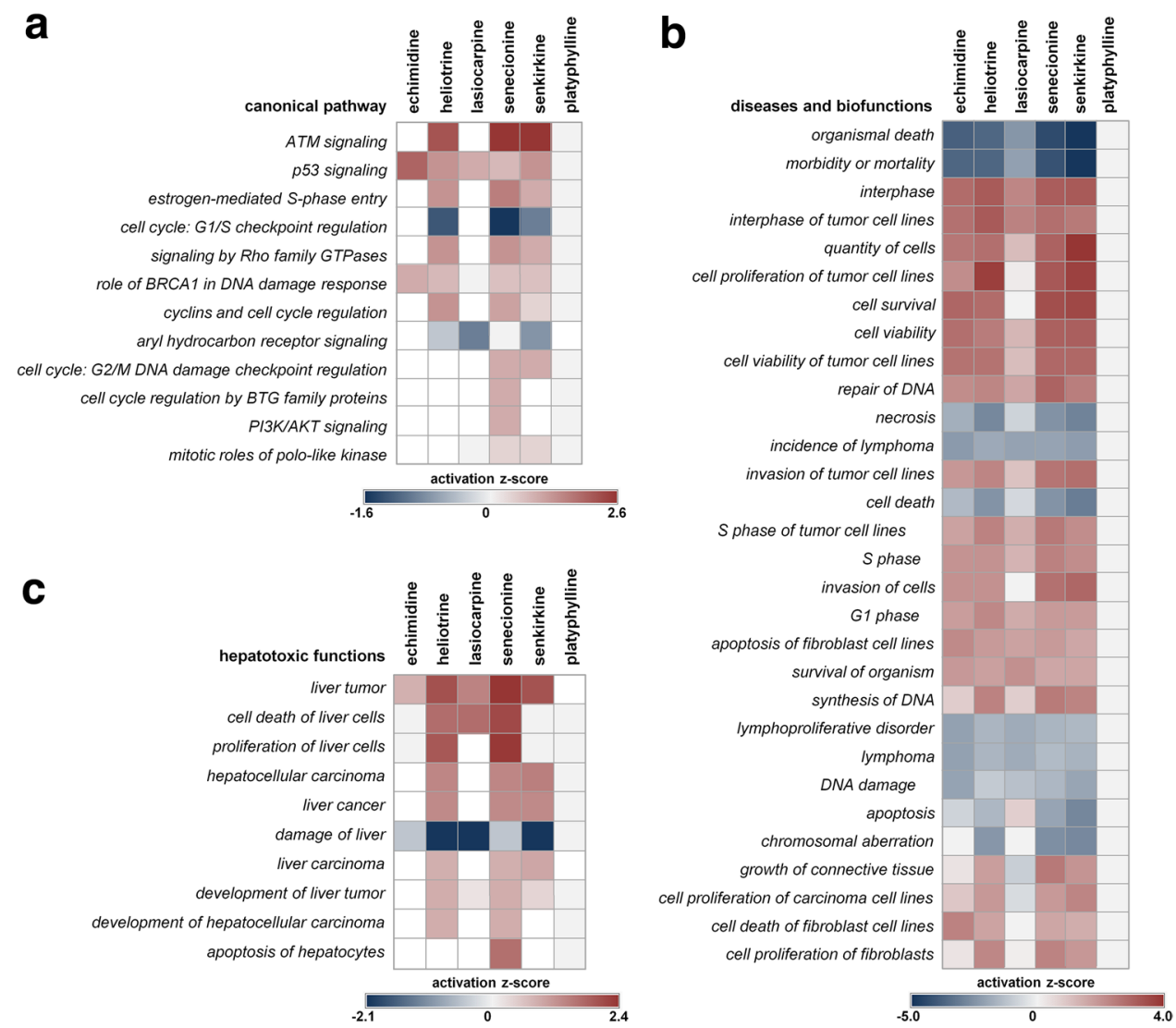

and senecionine), G1/S checkpoint regulation (inactivated by heliotrine, senkirkine and senecionine), cyclins and cell cycle regulation (activated by heliotrine, senkirkine and senecionine), G2/M DNA damage checkpoint regulation (activated by senkirkine and senecionine), cell cycle regulation by BTG family proteins (activated by senkirkine), and mitotic roles of polo-like kinase (activated by senkirkine and senecionine) were predicted to be regulated. All of these pathways are related to cell cycle regulation. PI3K/ AKT signaling was predicted to be activated by senkirkine. It regulates cell proliferation and cell death and is associated with cancer. Signaling by Rho family GTPases was predicted to be activated by heliotrine, senkirkine and senecionine. Rho family GTPases are important for regulating signal transduction and, thus, involved in several cellular processes. Finally, aryl hydrocarbon receptor signaling was predicted to be inactivated. The aryl hydrocarbon receptor is a transcription factor which regulates the expression of several genes, e.g., relevant for xenobiotic metabolism, cell proliferation and, thus, cell cycle regulation.

Most predictions in the area of diseases and functions are associated with cell viability/cell death/cell cycle, DNA damage or carcinogenesis (Fig. 5b). These predictions point to a genotoxic effect accompanied by replacement proliferation. Most of the predicted diseases and functions were affected by all PA except platyphylline. Platyphylline shows no impairment of any disease or biofunction.

Limiting the diseases and biofunctions predictions to hepatotoxicity only, resulted in predictions of functions related to liver cancer (Fig. 5c), such as stimulation of formation of liver tumor, hepatocellular carcinoma, liver cancer and liver carcinoma. Again, for platyphylline, no activation or inactivation of any hepatotoxic function was predicted.

IPA upstream analysis filtered for transcription regulators identified several regulators associated with cell cycle control (Fig. 6). Cyclin D1 (Ccnd1) is involved in G1/S transition during cell cycle control and was activated by all analyzed PA, except platyphylline. Ccnd1 interacts with retinoblastoma protein ( $\mathrm{Rb} / \mathrm{Rb} 1) . \mathrm{Rb} / \mathrm{Rb} 1$ inhibits cell cycle progression and functions as a tumor suppressor. $\mathrm{Rb} / \mathrm{Rb} 1$ was downregulated on mRNA level by all PA (except platyphylline) pointing again to proliferation and possibly a promotion of cancer development. Rb/Rb1 binds also to transcription factors of the E2f family leading to its inactivation. Transcription factors of the E2f family are also important for cell cycle progression. E2f1, E2f and E2f3 were predicted to be activated by all analyzed toxic PA. E2f6 was predicted to be inactivated by all PA, except lasiocarpine and platyphylline. Further activated transcription regulators, such as $\beta$-catenin, Foxm 1 , Irf1, 
Fig. 6 Transcription regulators predicted to be activated/inactivated by Ingenuity Pathway Analysis (IPA). A set of 284 significantly $(q<0.05)$ dysregulated rat liver transcripts was analyzed using IPA. Activation/ inactivation of upstream regulators, filtered for transcription regulators, is displayed without using cutoffs. The heatmap shows the activation $\mathrm{z}$-score for the respective regulator. The first 30 regulators with the highest positive or the lowest negative activation $\mathrm{z}$-score are shown

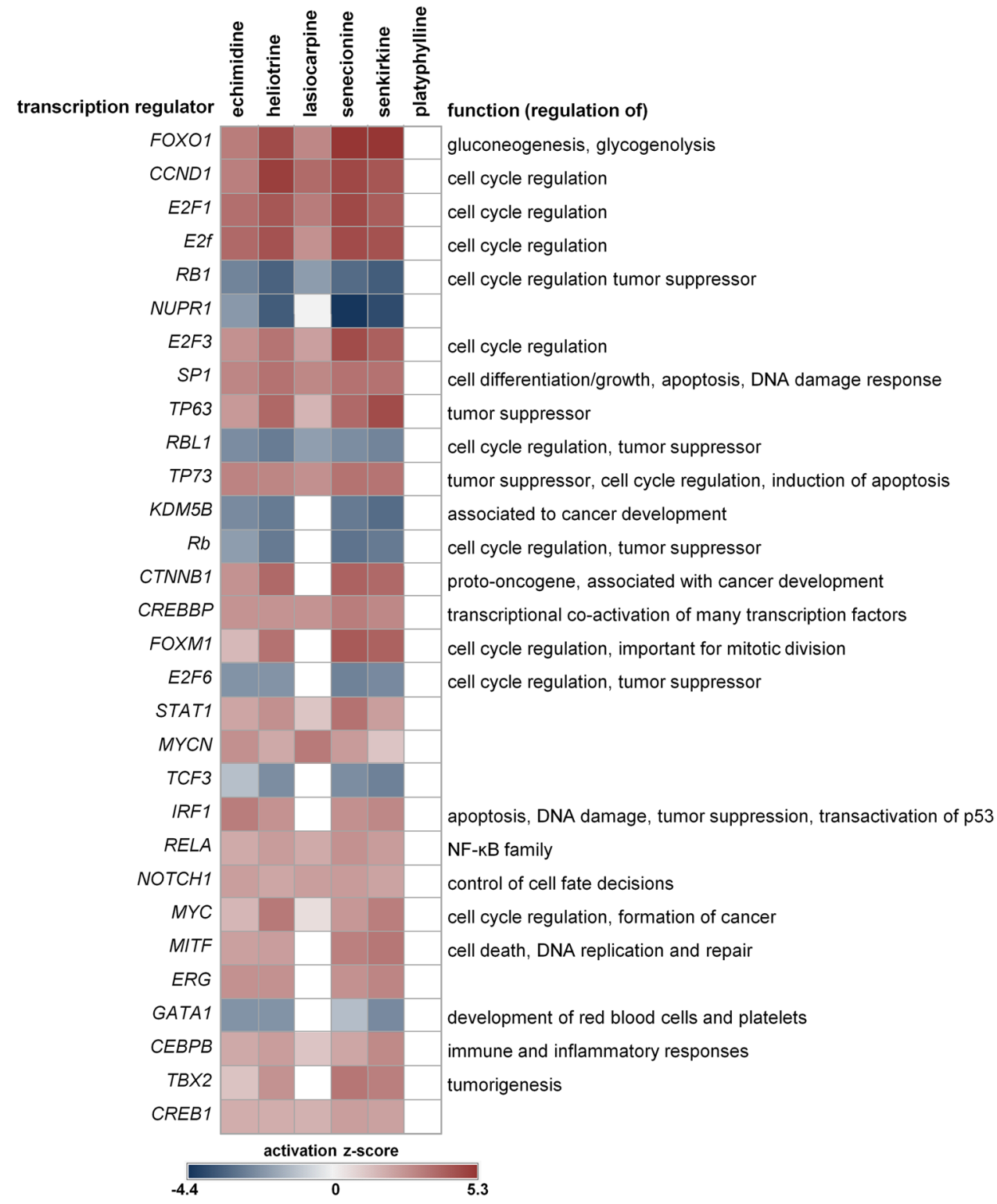

Myc, Mitf, Tbx2 with a somewhat lower z-score did also point to cell cycle regulation, DNA damage and cancer development.

\section{Discussion}

PA belong to the most widely distributed natural toxins known. However, there is still no in vivo study that includes human-relevant doses on the one hand and structure-dependent hepatotoxicity on the other. Thus, our transcriptomic study aimed to investigate pathways affected by PA in a subacute animal study using exposure scenarios relevant for highly exposed humans. It was of particular interest whether the different PA show the same toxicity pattern or result in activation/inactivation of specific, individual pathways.
Male Fischer rats were treated daily for 28 days orally with 6 structurally different PA and gene expression in the livers was analyzed using whole-genome microarrays. A daily dose of $3.3 \mathrm{mg} / \mathrm{kg}$ bw was administered as the highest dose to exclude unspecific effects resulting from general toxicity. The amount of this maximum dose results from a two-week and 13-week NTP study with the cyclic retronecine type diester riddelliine in Fischer rats (NTP 2003). According to the publication of Merz and Schrenk (2016) riddelliine exhibits comparable potency like senecionine which was used in our study. We decided to choose senecionine as representative of aforementioned PA group due to a better comparison to an existing set of in vitro and in vivo data (Hessel-Pras et al. 2020; Hessel et al. 2014; Luckert et al. 2015; Waizenegger et al. 2018). For comparing the highest dose used in our study to human exposure, human intake 
was estimated. Recently, a highly contaminated tea sample from the German retail market was found to contain $161 \mu \mathrm{g}$ PA per tea bag (Stiftung Warentest 2017). Excluding PA uptake from other sources, such as herbal spices or honey, an average $60-\mathrm{kg}$ adult who drinks 3 cups of this tea, would be exposed to $8-\mu \mathrm{g}$ PA $/ \mathrm{kg}$ bw. Therefore, the highest dose of $3.3 \mathrm{mg} / \mathrm{kg}$ bw used in the present study is about 400 times higher compared to the dose taken up by a human consuming 3 cups of the aforementioned contaminated tea; while, the lowest tested dose of $0.1 \mathrm{mg} / \mathrm{kg}$ bw is approximately 12.5-fold higher. A case report of infants describes that severe veno-occlusive disease was developed after ingestion of PA-contaminated food; the estimated daily PA uptake calculated from this report ranged from 0.8 to $3 \mathrm{mg} / \mathrm{kg}$ bw (Fox et al. 1978). The selected doses in this study, thus, represent a dose range that may be reached by human in worst case exposure scenarios. It should be noted that using higher doses of the compounds most probably would have provoked more pronounced transcriptional alterations. Nonetheless, this might have led to the masking of PA-specific effects by transcriptional signatures related to unspecific processes related to cell death and major organ damage. Therefore, the dosing was selected as described above, to allow for the detection of early transcriptional changes induced by PA.

Bioinformatic analysis identified a gene signature related to DNA damage, DNA repair, replacement proliferation/ impairment of cell cycle control. Also, the IPA analysis of diseases and functions, points to DNA repair, cell death and replacement proliferation. Upstream analysis filtered for transcription regulators identified additionally regulators that affect gene expression relevant for cell cycle progression/regulation, DNA damage response and cancer formation. Most of the identified dysregulated genes were affected by all 5 or at least 4 out of 5 toxic PA, indicating a similar mode of action. The genotoxic and carcinogenic potential of PA was already described in the literature. Merz and Schrenk (2016) furthermore list an overview about several studies analyzing DNA damage, chromosomal damage and mutagenic effects induced by different PA. However, our study is not based on a classic 2-year carcinogenicity study, but on a transcriptomics approach with comparably low doses of PA for a shorter period of 28 days. The results show that this approach identifies several dysregulated genes that play a role in response to DNA damage and carcinogenesis at dose levels that may occur in humans in worst case scenarios. Recently, a toxicogenomics directory of 162 rat hepatotoxicants has been established that also contains a list of genes most frequently dysregulated by genotoxic and non-genotoxic rat carcinogens (Grinberg et al. 2018). These genes (Table S5 in Grinberg et al. 2018) show a high degree of overlap with the 35 genes shown to be deregulated by all 5 PA in the present study (Fig. 3). Among genes in the overlap of the present study and the consensus signature of Grinberg et al (2018) are Aldhlal, Cdknla, Nr3c1, ERpl19, Mgmt, Fna, Clec4f, Ccng1, and Mybl2. No PA-induced effects were observed in the histopathological analysis; whereas, the transcriptomic approach showed clear alterations for the high-dose $(3.3 \mathrm{mg} / \mathrm{kg}$ bw) PA groups. The second highest dose $(1.0 \mathrm{mg} / \mathrm{kg}$ bw $)$ seemed to be in a borderline dose range where transcriptomics effects begin to be induced. Therefore, the results point to a higher sensitivity of a transcriptomics approach in comparison to conventional toxicity studies.

In our study, senecionine and senkirkine, both cyclic diesters of the retronecine or otonecine type, respectively, showed the strongest alterations compared to the vehicle control followed by heliotrine (monoester of the heliotridine type) and lasiocarpine (non-cyclic diester of the heliotridine type). Echimidine (non-cyclic diester of the retronecine type) fluctuated in the range of heliotrine and lasiocarpine. Senecionine was identified before to belong to the most potent PA. Waizenegger et al. (2018) compared the cytotoxic and apoptotic potential of the four PA echimidine, heliotrine, senecionine and senkirkine in vitro in 24-h and 14-day exposure scenarios. Senecionine showed the strongest effects followed by echimidine. Heliotrine and senkirkine showed much weaker effects. Louisse et al. (2019) investigated the genotoxic potential of 37 structurally different PA by analyzing the phosphorylation of the histone H2AX. According to their results, senecionine, lasiocarpine and echimidine show the highest toxic potential, followed by senkirkine and finally heliotrine. Merz and Schrenk (2016) suggested the introduction of relative potency factors for PA risk assessment. Their relative interim potency factors (iRPF) are based on the combined genotoxic potency in Drosophila, cytotoxic potency in vitro and acute toxicity in adult rodents (i.p./i.v. injection). According to Merz and Schrenk, the PA senecionine, senkirkine and lasiocarpine belong to the most potent PA (iRPFs of 1.0), followed by heliotrine (iRPF 0.3) and echimidine (iRPF 0.1). The observations made in our study are very much in line with the suggested iRPFs. Some differences were observed for lasiocarpine, which showed a lower number of dysregulated genes compared to senecionine, senkirkine, echimidine and heliotrine. Compared to the results of Louisse et al. (2019), our results show a higher toxic potential for senkirkine and a lower toxic potential for echimidine and lasiocarpine. However, differences in potency can be due to the different test systems or exposure routes in the different studies. For the strong hepatotoxicity of PA, bioavailability and toxicokinetic properties most likely play a key role. Thus, our study compares for the first time the induction of hepatotoxicity by six structurally different PA after an oral exposure representing the real exposure of humans. A different intestinal first pass effect and consequently different levels of liver toxicity can be assumed due to a different passage rate over the intestinal 
barrier which was shown in vitro for four structurally different PA (Hessel et al. 2014). In Caco-2 cells, echimidine exhibited the lowest passage rate over the intestinal monolayer due to an ABCB1-driven efflux. Due to structural similarities between echimidine and lasiocarpine (both openchain diester), the reduction of oral bioavailability by active efflux mechanisms can be hypothesized.

The analyzed gene signature points to DNA-damaging properties accompanied by replacement proliferation and cancer development. All analyzed hepatotoxic PA induced similar pathways. The effect strength depended on the respective PA structure type. Senecionine and senkirkine, both cyclic diesters of the retronecine and otonecine type, respectively, showed the strongest effect, followed by the non-cyclic diester of the retronecine type, echimidine, and the monoester of the heliotridine type, heliotrine. Lasiocarpine, a non-cyclic diester of the heliotridine type, induced comparably weak effects. Platyphylline, a PA representative assumed to be non-hepatotoxic, induced no relevant gene expression alterations, as expected. The detection of the DNA-damaging properties without observing histopathological changes (dose $1.0 \mathrm{mg}$ PA per $\mathrm{kg} \mathrm{bw}$ ) and treatment duration far below classic carcinogenicity studies suggests that transcriptomic approaches may be a helpful tool to investigate the genotoxic potential of possible toxins. While omics data represent promising approaches for the early, sensitive detection of certain toxic effects which become histopathologically detectable much later, there are still several obstacles which impede the use of omics data in a regulatory context. This includes, for example, harmonization of technology platforms and data analysis workflows, as well as the definition of adversity (Marx-Stoelting et al. 2015). Future research is, thus, still needed to facilitate the implementation of omics data in regulatory toxicology.

Acknowledgements Open Access funding provided by Projekt DEAL. This work was supported by the German Federal Institute for Risk Assessment (grant numbers 1329-554, 1322-591, 1322-624). Ramonique Lim and Laura Wittek are acknowledged for their support with microarray verification. We also thank Prof. Dr. Jörg Rahnenführer and Dr. Birte Hellwig for their support with the statistical analysis.

\section{Compliance with ethical standards}

Conflict of interest The authors declare that there are no conflicts of interest.

Open Access This article is licensed under a Creative Commons Attribution 4.0 International License, which permits use, sharing, adaptation, distribution and reproduction in any medium or format, as long as you give appropriate credit to the original author(s) and the source, provide a link to the Creative Commons licence, and indicate if changes were made. The images or other third party material in this article are included in the article's Creative Commons licence, unless indicated otherwise in a credit line to the material. If material is not included in the article's Creative Commons licence and your intended use is not permitted by statutory regulation or exceeds the permitted use, you will need to obtain permission directly from the copyright holder. To view a copy of this licence, visit http://creativecommons.org/licenses/by/4.0/.

\section{References}

Benjamini Y, Hochberg Y (1995) Controlling the false discovery rate: a practical and powerful approach to multiple testing. J Roy Stat Soc: Ser B (Methodol) 57(1):289-300

Bergmeyer HU (1983) Methods of enzymatic analysis, vol 3. Verlag Chemie, Weinheim

BfR (2018) Aktualisierte Risikobewertung zu Gehalten an 1,2-ungesättigten Pyrrolizidinalkaloiden (PA) in Lebensmitteln. BfR Opinion No 020(2018):1-13

BfR (2019) Pyrrolizidinalkaloidgehalt in getrockneten und tiefgefrorenen Gewürzen und Kräutern zu hoch Stellungnahme $\mathrm{Nr}$ 017/2019 des BfR vom 13 Mai 1-16

Bodi D, Ronczka S, Gottschalk C et al (2014) Determination of pyrrolizidine alkaloids in tea, herbal drugs and honey. Food Addit Contam Part A 31(11):1886-1895. https://doi. org/10.1080/19440049.2014.964337

Bras G, Jelliffe DB, Stuart KL (1954) Veno-occlusive disease of liver with nonportal type of cirrhosis, occurring in Jamaica. AMA Arch Pathol 57(4):285-300

Culvenor CCJ, Downing DT, Edgar JA, Jago MV (1969) Pyrrolizidine alkaloids as alkylating and antimitotic agents. Ann N Y Acad Sci 163(2):837

Datta DV, Khuroo MS, Mattocks AR, Aikat BK, Chhuttani PN (1978) Herbal medicines and veno-occlusive disease in India. Postgrad Med J 54(634):511-515. https://doi.org/10.1136/ pgmj.54.634.511

Edgar R, Domrachev M, Lash AE (2002) Gene Expression Omnibus: NCBI gene expression and hybridization array data repository. Nucleic Acids Res 30(1):207-210. https://doi.org/10.1093/ nar/30.1.207

EFSA (2017) Risks for human health related to the presence of pyrrolizidine alkaloids in honey, tea, herbal infusions and food supplements - EFSA panel on contaminants in the food chain (CONTAM). EFSA J 15(7):4908. https://doi.org/10.2903/j. efsa.2017.4908

Fox DW, Hart MC, Bergeson PS, Jarrett PB, Stillman AE, Huxtable RJ (1978) Pyrrolizidine (Senecio) intoxication mimicking Reye syndrome. J Pediatr 93(6):980-982

Fu PP, Xia Q, Lin G, Chou MW (2004) Pyrrolizidine alkaloids - genotoxicity, metabolism enzymes, metabolic activation, and mechanisms. Drug Metab Rev 36(1):1-55. https://doi.org/10.1081/ dmr-120028426

Grinberg M, Stober RM, Albrecht W et al (2018) Toxicogenomics directory of rat hepatotoxicants in vivo and in cultivated hepatocytes. Arch Toxicol 92(12):3517-3533. https://doi.org/10.1007/ s00204-018-2352-3

Hessel-Pras S, Braeuning A, Guenther G et al (2020) The pyrrolizidine alkaloid senecionine induces CYP-dependent destruction of sinusoidal endothelial cells and cholestasis in mice. Arch Toxicol 94(1):219-229. https://doi.org/10.1007/s00204-019-02582-8

Hessel S, Gottschalk C, Schumann D, These A, Preiss-Weigert A, Lampen A (2014) Structure-activity relationship in the passage of different pyrrolizidine alkaloids through the gastrointestinal barrier: ABCB1 excretes heliotrine and echimidine. Mol Nutr Food Res 58(5):995-1004. https://doi.org/10.1002/mnfr.201300707

Kakar F, Akbarian Z, Leslie T et al (2010) An outbreak of hepatic veno-occlusive disease in Western Afghanistan associated with 
exposure to wheat flour contaminated with pyrrolizidine alkaloids. J Toxicol. https://doi.org/10.1155/2010/313280

Louisse J, Rijkers D, Stoopen G et al (2019) Determination of genotoxic potencies of pyrrolizidine alkaloids in HepaRG cells using the gammaH2AX assay. Food Chem Toxicol 131:110532. https ://doi.org/10.1016/j.fct.2019.05.040

Luckert C, Hessel S, Lenze D, Lampen A (2015) Disturbance of gene expression in primary human hepatocytes by hepatotoxic pyrrolizidine alkaloids: A whole genome transcriptome analysis. Toxicol In Vitro 29(7):1669-1682. https://doi.org/10.1016/j. tiv.2015.06.021

Marx-Stoelting P, Braeuning A, Buhrke T et al (2015) Application of omics data in regulatory toxicology: report of an international BfR expert workshop. Arch Toxicol 89(11):2177-2184. https:// doi.org/10.1007/s00204-015-1602-x

Mattocks AR (1986) Chemistry and toxicology of pyrrolizidine alkaloids. Academic Press, London

Merz KH, Schrenk D (2016) Interim relative potency factors for the toxicological risk assessment of pyrrolizidine alkaloids in food and herbal medicines. Toxicol Lett 263:44-57. https://doi. org/10.1016/j.toxlet.2016.05.002

Mohabbat O, Shafiq Younos M, Merzad AA, Srivastava RN, Ghaos Sediq G, Aram GN (1976) An outbreak of hepatic veno-occlusive disease in North-Western Afghanistan. The Lancet 308(7980):269-271. https://doi.org/10.1016/S0140 -6736(76)90726-1

Moreira R, Pereira DM, Valentão P, Andrade PB (2018) Pyrrolizidine alkaloids: chemistry, pharmacology, toxicology and food safety. Int J Mol Sci 19(6):1668. https://doi.org/10.3390/ijms19061668

Mulder PPJ, López P, Castelari M et al (2018) Occurrence of pyrrolizidine alkaloids in animal- and plant-derived food: results of a survey across Europe. Food Addit Contam Part A 35(1):118-133. https://doi.org/10.1080/19440049.2017.1382726

Musa J, Aynaud M-M, Mirabeau O, Delattre O, Grünewald TGP (2017) MYBL2 (B-Myb): a central regulator of cell proliferation, cell survival and differentiation involved in tumorigenesis. Cell Death Amp Dis 8:e2895. https://doi.org/10.1038/cddis.2017.244

National Institutes of Health (1993) NTP Technical report on toxicity studies of riddelliine administered by gavage to $\mathrm{F} 344 / \mathrm{N}$ rats and B6C3P1 mice. NIH Publication No 94-3350

NTP (2003) Toxicology and carcinogenesis studies of riddelliine (CAS No 23246-96-0) in F344/N rats and B6C3F1 mice (gavage studies). Nation Toxicol Program Techn Rep Ser (508):1-280

R Core Team (2017) A language and environment for statistical computing. R Foundation for Statistical Computing. Vienna, Austria https://www.R-project.org/
Rasinger J, Lie KK Understanding the molecular mechanisms of contaminant exposures in fish and their interaction with nutrients. In

Ritchie ME, Phipson B, Wu D et al (2015) limma powers differential expression analyses for RNA-sequencing and microarray studies. Nucleic Acids Res 43(7):e47. https://doi.org/10.1093/nar/gkv007

Rozen S, Skaletsky H (2000) Primer3 on the WWW for general users and for biologist programmers. Methods Molecular Biol (Clifton, NJ) 132:365-386

Ruan J, Yang M, Fu P, Ye Y, Lin G (2014) Metabolic activation of pyrrolizidine alkaloids: Insights into the structural and enzymatic basis. Chem Res Toxicol 27(6):1030-1039. https://doi. org/10.1021/tx500071q

Sinha SK, Chaudhary PM (2004) Induction of apoptosis by X-linked ectodermal dysplasia receptor via a caspase 8-dependent mechanism. J Biol Chem 279(40):41873-41881. https://doi.org/10.1074/ jbc.M407363200

Smyth GK, Ritchie M, Thorne N, Wettenhall J, Hu Y (2019) Limma: Linear Models for Microarray and RNA-Seq Data User's Guide. https://bioconductororg/packages/release/bioc/vignettes/limma/ inst/doc/usersguide.pdf

Stegelmeier BL, Edgar JA, Colegate SM et al (1999) Pyrrolizidine alkaloid plants, metabolism and toxicity. J Nat Toxins 8(1):95-116

Stiftung Warentest (2017) Kamillentee von Kusmi: Extrem mit Schadstoffen belastet. https://www.testde/Kamillentee-von-Kusmi-Extre m-mit-Schadstoffen-belastet-5126437-0/ Accessed date 19 March 2019

Tandon BN, Tandon HD, Tandon RK, Narndranathan M, Joshi YK (1976) An epidemic of veno-occlusive disease of liver in Central India. The Lancet 308(7980):271-272. https://doi.org/10.1016/ S0140-6736(76)90727-3

Waizenegger J, Braeuning A, Templin M, Lampen A, Hessel-Pras S (2018) Structure-dependent induction of apoptosis by hepatotoxic pyrrolizidine alkaloids in the human hepatoma cell line HepaRG: Single versus repeated exposure. Food Chem Toxicol 114:215226. https://doi.org/10.1016/j.fct.2018.02.036

Wiedenfeld H (2011) Plants containing pyrrolizidine alkaloids: toxicity and problems. Food Addit Contam Part A 28(3):282-292. https:// doi.org/10.1080/19440049.2010.541288

Wiedenfeld H, Roeder E, Bourauel T, Edgar J (2008) Pyrrolizidine alkaloids - structure and toxicity. Verlag V\&R Unipress, Bonn

Publisher's Note Springer Nature remains neutral with regard to jurisdictional claims in published maps and institutional affiliations. 